\title{
A CONDITIONAL STOCHASTIC PROJECTION METHOD APPLIED TO A PARAMETRIC VIBRATIONS PROBLEM
}

\author{
Wlodzimierz BRZAKALA, Aneta HERBUT \\ Faculty of Civil Engineering, Wroclaw University of Technology, Poland \\ Received 31 Oct 2013; accepted 09 Jan 2014
}

\begin{abstract}
Parametric vibrations can be observed in cable-stayed bridges due to periodic excitations caused by a deck or a pylon. The vibrations are described by an ordinary differential equation with periodic coefficients. The paper focuses on random excitations, i.e. on the excitation amplitude and the excitation frequency which are two random variables. The excitation frequency $\omega_{\mathrm{L}}$ is discretized to a finite sequence of representative points, $\omega_{\mathrm{L}, i}$. Therefore, the problem is (conditionally) formulated and solved as a one-dimensional polynomial chaos expansion generated by the random excitation amplitude. The presented numerical analysis is focused on a real situation for which the problem of parametric resonance was observed (a cable of the Ben-Ahin bridge). The results obtained by the use of the conditional polynomial chaos approximations are compared with the ones based on the Monte Carlo simulation (truly two-dimensional, not conditional one). The convergence of both methods is discussed. It is found that the conditional polynomial chaos can yield a better convergence then the Monte Carlo simulation, especially if resonant vibrations are probable.
\end{abstract}

Keywords: stochastic dynamics, parametric vibrations, Hermite's polynomial chaos, Monte Carlo simulation.

\section{Introduction}

A dangerous phenomenon of parametric resonances can be observed among others in bridge cables during a strong wind gusts and sometimes due to a traffic load. It happens due to periodic movements of a cable support, which are usually caused by deck or rarely by pylon vibrations. There are several well-documented case studies in which parametric vibrations were observed in bridge cables, like Guazu Bridge in Argentina, Belgian bridges Ben-Ahin and Wandre, Annacis Bridge in Canada, Faro Bridge in Denmark, Helgoland Bridge in Norway, Erasmus Bridge (Netherlands), Burlington (USA) or Second Severn Crossing (GB) Bridges. In each of the reported cases, the amplitude of vibrations increased exponentially; that is a very specific feature of parametric resonant vibrations which allows to distinguish this phenomenon from typical resonant vibrations with the amplitude increasing linearly (in absence of damping). Therefore, parametric resonant vibrations are much more dangerous than standard load-excited resonances. Moreover, parametric vibrations appear usually when an excitation frequency is near to doubled eigenfrequency - the instability zone is the largest in that case (Nayfeh, Mook 1995).

Relevant case studies of structures and the observed parametric vibrations are presented in Caetano (2007), Lilien and Pinto Da Costa (1994), Royer-Carafangi (2003). The presented paper focuses on a linear differential equation with random periodic coefficients. The Hermite-type polynomial chaos expansion is supported by the stochastic projection method (the Galerkin approach) and is implemented to a case of two input random variables. The convergence of results is presented in a context of the Monte Carlo simulation.

\section{Polynomial chaos approximation - theoretical background, literature review}

Generally, probabilistic analyses can be classified into two categories: methods using a statistical approach and methods based on a non-statistical approach. The statistical approach includes first of all the widely used Monte Carlo simulation (MC). Statistical methods are easy to implement but sometimes - in cases of complicated mathematical models - not efficient. Therefore, research effort has been made in developing non-statistical methods, like for instance perturbation techniques, usually addressed to "small uncertainties". Ghanem and Spanos proposed an innovative approach called polynomial chaos method (PC) and applied it successfully to various problems of mechanics (Ghamen, Spanos 1991); it is a situation where a stochastic process with finite variance can be presented in form of infinite convergent series, based on polynomials of standard Gaussian variable $\xi$ (with zero mean value and standard deviation equal to 1 ).

The PC method separates stochastic components from deterministic ones for both - input data and random solutions. Random components of solutions can be calculated independently from the deterministic ones. If a random variable is a parameter taken into consideration, it 
can be expressed as a series of polynomials of the standard Gaussian variable (or variables) with series coefficients which are real numbers. For stochastic processes, the series of polynomials of the standard Gaussian variable are similar as for random variables, but the coefficients are time-dependent functions. The same is true for random fields but the coefficients are position- and/or timedependent. Clearly, some regularity conditions are required for both data and solutions, usually not restrictive in engineering applications.

The year 1991 - when Ghanem and Spanos applied the PC in conjunction with the finite element method (Ghamen, Spanos 1991) - was the turning point in the method development. In recent years, the PC method has been applied to a wide spectrum of stochastic problems, for instance to the theory of elasticity and plasticity (Ghamen, Spanos 1991; Anders, Hori 1999), stochastic dynamics (Ghanem, Spanos 1993; Sarkar, Ghanem 2002), wave propagation in random media (Manolis, Karakostas 2003), stochastic Stokes equations (Qu, Xu 2013) or sensitivity analysis (Sudret 2008; Crestaux et al. 2009; Blatman, Sudret 2010). In the mentioned examples, the application of the PC expansion presents the evidence of a good calculation efficiency. It refers also to relatively large random fluctuations, far beyond the scope of the simplest perturbation techniques. The success of this approach yields also from a conjunction with the Galerkin approximation, i.e. the best (least square) approximation in abstract Hilbert spaces.

Traditionally (Ghamen, Spanos 1991), the PC representations are based on the Hermite polynomials of the standard Gaussian variable $\xi$. However, in the case of complex nonlinear problems and non-Gaussian distributions of the input data or the system response, it is better to replace the Hermite polynomials by a more suitable function base. If the input data is very close to the Gaussian random variable then the linear approximation (with only two terms) can be accepted but the response expansion is usually much longer; and opposite, a change of the function base can simplify the response approximation though the simple Gaussian input will have a less accurate polynomial approximation. In other words, the "optimal" selection of the function base is problem-dependent. The so called generalized polynomial chaos expansions are addressed by Xiu and Karniadakis (2002a, 2002b, 2003), Le Maitre et al. (2004) and others. In recent years, the generalized polynomial chaos was successfully applied to solve the problems of diffusion and heat flow (Xiu, Karniadakis 2002a, 2003), or problems connected with interaction between structure and fluid (Xiu, Karniadakis 2002b; Lucor, Karniadakis 2004), etc.

Recently, a new insight into the background of the PC method was presented by Wan and Karniadakis $(2005,2006)$. The authors formulate a so called multielement generalized polynomial chaos method to deal with long-term integration and discontinuities in stochastic differential equations. On the other hand, Blatman and Sudret proposed the so called sparse polynomial chaos approach (Blatman, Sudret 2010, 2011). To decrease the computational cost of classical PC schemes in the case of large size of the input random vector, the authors presented a method that builds a sparse PC expansion.

\section{Fundamentals of the polynomial chaos approximation}

\subsection{A stochastic scalar product}

Consider a sequence of uncorrelated random variables $B_{k}(\gamma)$, which have zero expected values and finite variances: $\quad \mathrm{E}\left[B_{k}(\gamma)\right]=0, \quad \operatorname{Var}\left[B_{k}(\gamma)\right]<+\infty, \quad$ for $k=1,2, \ldots, n \leq+\infty$, and assume that $B_{0}=$ const $=1$. It is a well-established fact that the expected value operator E[.] defines a scalar product $\langle. .$.$\rangle as follows:$ $\left\langle B_{i}, B_{j}\right\rangle \stackrel{\text { def }}{=} \mathrm{E}\left[B_{i} \cdot B_{j}\right]$. The lack of correlation yields the orthogonality condition of the random variables $B_{k}(\gamma)$, i.e. $\left\langle B_{i}, B_{j}\right\rangle=0$, if and only if $i \neq j$.

A special selection of random variables $B_{k}(\gamma)$, which introduce the stochastic basis, makes it possible to expand every finite-variance random variable $X(\gamma)$ in a series (Ghamen, Spanos 1991):

$$
X(\gamma)=\sum_{k=0}^{n \leq \infty} a_{k} \cdot B_{k}(\gamma)
$$

where: $\mathrm{E}[X]=a_{0}$,

$$
\begin{aligned}
\operatorname{Var}[X]= & \mathrm{E}\left[\left(X-a_{0}\right)^{2}\right]=\mathrm{E}\left[\left(\sum_{i=1}^{n} a_{i} \cdot B_{i}\right) \cdot\left(\sum_{j=1}^{n} a_{j} \cdot B_{j}\right)\right]= \\
& \sum_{i=1}^{n} \sum_{j=1}^{n} a_{i} \cdot a_{j} \cdot\left\langle B_{i}, B_{j}\right\rangle=\sum_{k=1}^{n}\left(a_{k}\right)^{2} \cdot\left\langle B_{k}, B_{k}\right\rangle<+\infty .
\end{aligned}
$$

\subsection{The stochastic projection method}

The stochastic projection method is the best mean-square approximation based on the distance defined by the scalar product. Usually, explicit form of the random variable $X$ is unknown thus it is recommended to use its approximation $X^{(m)}$ in the form of the finite expansion (1), so for $m<n \leq \infty$.

It is a general fact that the best mean-square $m$-dimensional approximation of $X$ coincides with $m$-truncation of the orthogonal expansion (1). Indeed, for:

$$
X^{(m)}=\sum_{k=0}^{m} \alpha_{k} \cdot B_{k},
$$

the mean-square distance:

$$
\mathrm{d}\left(X^{(m)}, X\right) \stackrel{\operatorname{def}}{=} \sqrt{\left\langle\left(X^{(m)}-X\right)\left(X^{(m)}-X\right)\right\rangle}
$$

can be calculated from the following expression: 


$$
\begin{aligned}
\mathrm{d}\left(X^{(m)}, X\right)^{2}= & \sum_{k=0}^{m}\left(\alpha_{k}-a_{k}\right)^{2} \cdot\left\langle B_{k}, B_{k}\right\rangle+ \\
& \sum_{k=m+1}^{n}\left(a_{k}\right)^{2} \cdot\left\langle B_{k}, B_{k}\right\rangle \rightarrow \min ,
\end{aligned}
$$

which becomes minimal just for $\alpha_{k}=a_{k}$,

$k=0,1,2, \ldots, m<n \leq+\infty$.

\subsection{The Hermite polynomials of the Gaussian random variable}

The traditional Hermite polynomials $\mathrm{He}_{k}(x)$ of the real variable $x$ are orthogonal with the standard Gaussian density $\varphi(x)$ taken as the weight function:

$$
\begin{aligned}
& \int_{-\infty}^{+\infty} \mathrm{He}_{i}(x) \cdot \mathrm{He}_{j}(x) \cdot \phi(x) \mathrm{d} x= \\
& 1 / \sqrt{2 \pi} \int_{-\infty}^{+\infty} \mathrm{He}_{i}(x) \cdot \mathrm{He}_{j}(x) \cdot \exp \left(-x^{2} / 2\right) \mathrm{d} x=i ! \cdot \delta_{i j},
\end{aligned}
$$

where $\delta_{i j}$ is the Kronecker delta for $i, j \geq 0$.

The formula (4) is a simple prototype for the stochastic orthogonality condition, if the stochastic basis is introduced as $B_{k}(\gamma) \stackrel{\text { def }}{=} \mathrm{He}_{k}(\xi(\gamma))$ where $\xi \in \mathrm{N}(0 ; 1)$, i.e. $\xi$ is a standard Gaussian random variable, $\mathrm{E}[\xi]=0$, $\operatorname{Var}[\xi]=1$. This happens because - by definition - for such stochastic basis:

$$
\begin{aligned}
& \left\langle B_{i}(\gamma), B_{j}(\gamma)\right\rangle \stackrel{\text { def }}{=} \mathrm{E}\left[B_{i}(\xi) \cdot B_{j}(\xi)\right]= \\
& \mathrm{E}\left[\operatorname{He}_{i}(\xi) \cdot \mathrm{He}_{j}(\xi)\right]=\int_{-\infty}^{+\infty} \operatorname{He}_{i}(x) \cdot \mathrm{He}_{j}(x) \cdot \phi(x) \mathrm{d} x .
\end{aligned}
$$

Estimation of the approximation error (and the convergence rate) is strongly influenced by the weight function $\varphi(x)$. The stochastic Hermite approximation is reliable for "small" values of $|\xi|$ and is less reliable for "large" fluctuations - which are, however, less probable in case of the random variable $\xi \in \mathrm{N}(0 ; 1)$.

\section{Application of the polynomial chaos approximation}

A PC-implementation is presented for a differential equation with periodic coefficients which yields from the equation of motion.

\subsection{Equation of motion of a bridge cable with stochastic excitation}

Transversal vibrations $w(x, t)$ of a cable, i.e. in the $y$-direction in Figure 1, due to a vertical kinematic excitation $\Delta(t)$, are the physical prototype of the mathematical model. In the considered example the excitation is applied only in form of kinematic excitation with in-plane

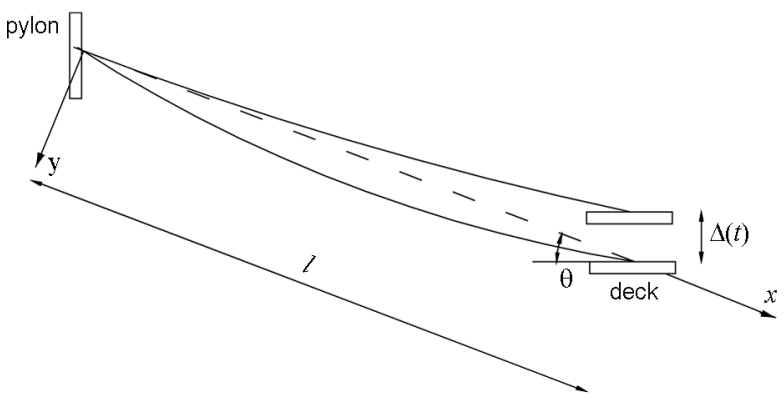

Fig. 1. Bridge cable displacement due to deck movements $\Delta(t)$

components. For that case, only in-plane cable vibrations occur. That is why only a two-dimensional dynamic problem is considered in the paper. The equation of motion (6) is derived in Brzakala (2013):

$H_{\mathrm{st}} \cdot \frac{\partial^{2} w(x, t)}{\partial x^{2}}+\frac{E P}{l} \cdot\left(\frac{\partial^{2} w(x, t)}{\partial x^{2}}+\frac{\mathrm{d}^{2} z(x)}{\mathrm{d} x^{2}}\right)$.
$\int_{0}^{l}\left(\frac{\partial w(x, t)}{\partial x} \cdot \frac{\mathrm{d} z(x)}{\mathrm{d} x}+\frac{1}{2} \cdot\left(\frac{\partial w(x, t)}{\partial x}\right)^{2}\right) \mathrm{d} x+\frac{E P}{l}$.

$\Delta(t) \cdot \sin \theta \cdot\left(\frac{\partial^{2} w(x, t)}{\partial x^{2}}+\frac{\mathrm{d}^{2} z(x)}{\mathrm{d} x^{2}}\right)-$

$c_{w} \cdot \frac{\partial w(x, t)}{\partial t}-m \cdot \frac{\partial^{2} w(x, t)}{\partial t^{2}}=0$,

where: $E$ - the Young modulus of the cable; $P$ - crosssectional area of the cable; $m$ - cable mass per unit length; $c_{w}$ - damping coefficient for the transversal vibrations $c_{w}=2 \cdot m \cdot \omega_{1} \cdot \xi ; \omega_{1}-$ natural eigenfrequency for the first symmetric mode of the cable in the transverse direction, for small values of the Irvine parameter it can be assumed as $\omega_{1}=\pi / l \cdot \sqrt{S / m}$, like for the taut string (Irvine 1981; Caetano 2007); $\xi$ - fraction of the critical damping; $z(x)$ - assumed function of a sag for the inclined cable in case of the static load (dead weight of the cable as well as an initial tension force) $z(x)=m \cdot g \cdot l^{2} \cdot \cos \theta /(2 \cdot S) \cdot(x / l) \cdot(1-(x / l)) \quad$ (Zhou et al. 2006); $S$ - is an initial tension force in the cable; $H_{\text {st }}$ - static component of an axial force in the cable (in $x$-direction), $H_{\text {st }}=S=$ const. $; g-$ is the gravity constant $g=9.81 \mathrm{~m} / \mathrm{s}^{2}$.

The kinematic excitation $\Delta(t)=\Delta \cdot \cos \left(\omega_{\mathrm{L}} t\right)$ is the reason of periodic changes of coefficients. Positiondependent function $z(x)$ and $H_{\text {st }}$ are known (pre-defined). The solution $w(x, t)$ is assumed in the separated variables, see Georgakis and Taylor (2005), as the following approximation:

$$
w(x, t)=w(t) \cdot \sin (\pi \cdot x / l)+\Delta(t) \cdot \cos \theta \cdot(x / l)^{2} .
$$

The second component in the formula (7) can be also assumed as a linear function, not the parabolic one 
(El Ouni, Ben Kahla 2012). However, Georgakis and Taylor propose the parabolic approximation which involves the sag changes due to a deck movement (Georgakis, Taylor 2005). Note that both concepts are approximate.

The single degree of freedom model is considered because for cables with a small sag, excited only parametrically, it is impossible to get complex vibrations, i.e. the ones consisting of several forms; so called combination resonance does not appear (Takahashi 1991). Selection of the excitation frequencies should be such that the parametric vibrations with the first form appear. They are close to the doubled first eigenfrequency (Nayfeh, Mook 1995). To get other forms of vibrations, higher excitation frequencies are necessary.

Probabilistic analyses of the random response of the system due to random fluctuations of the kinematic excitation (two random variables $\Delta$ and $\omega_{\mathrm{L}}$ ) are the objective of the paper. The PC expansion and the MC simulation are applied to solve this problem.

Practically justified cases of small displacements are considered, so nonlinear terms in equation of motion are neglected. Due to the approximation (7) the equation of motion (6) for the point located in the middle of the cable span, $x=l / 2$, can be written in form of the following ordinary differential equation:

$$
\begin{aligned}
& S_{1} \cdot \frac{\mathrm{d}^{2}}{\mathrm{~d} t^{2}} w(t)+S_{2} \cdot \frac{\mathrm{d}}{\mathrm{d} t} w(t)+ \\
& \left(S_{3}+S_{4} \cdot \Delta(t)+S_{5} \cdot \Delta(t)^{2}\right) \cdot w(t)+S_{6} \cdot \Delta(t)^{3}+ \\
& S_{7} \cdot \Delta(t)^{2}+S_{8} \cdot \frac{\mathrm{d}^{2}}{\mathrm{~d} t^{2}} \Delta(t)+S_{9} \cdot \frac{\mathrm{d}}{\mathrm{d} t} \Delta(t)+S_{10} \cdot \Delta(t)=0,
\end{aligned}
$$

where: $\Delta(t)=\Delta \cdot \cos \left(\omega_{\mathrm{L}} t\right)$ is a kinematic excitation due to the deck movement; $S_{i}$ - ten deterministic constants depending on geometrical or material parameters of the cable:

$$
\begin{aligned}
S_{1}= & m, S_{2}=c_{w}, \\
S_{3}= & S \cdot \pi^{2} / l^{2}+0.637 \cdot E \cdot P \cdot g^{2} \cdot m^{2} \cdot \cos ^{2} \theta / S^{2}, \\
S_{4}= & E \cdot P \cdot \sin \theta \cdot \pi^{2} / l^{3}-4.191 \cdot E \cdot P \cdot g \cdot m \cdot \cos ^{2} \theta /\left(l^{2} \cdot S\right), \\
S_{5}= & 9.126 \cdot E \cdot P \cdot \cos ^{2} \theta / l^{4}, \\
S_{6}= & -1.333 \cdot E \cdot P \cdot \cos ^{3} \theta / l^{4}, \\
S_{7}= & E \cdot P \cdot g \cdot m \cdot \cos ^{3} \theta /\left(l^{2} \cdot S\right)-2 \cdot E \cdot P \cdot \cos \theta \cdot \sin \theta / l^{3}, \\
S_{8}= & -0.250 \cdot m \cdot \cos \theta, S_{9}=-0.250 \cdot c_{w} \cdot \cos \theta, \\
S_{10}= & E \cdot P \cdot g \cdot m \cdot \cos \theta \cdot \sin \theta /(S \cdot l)- \\
& 0.167 \cdot E \cdot P \cdot g^{2} \cdot m^{2} \cdot \cos ^{3} \theta / S^{2}-2 \cdot S \cdot \cos \theta / l^{2} .
\end{aligned}
$$

\subsection{Example - deterministic and random variables}

The deterministic input parameters are presented in Table 1 .

Only positive values of the excitation amplitude and positive frequencies are possible, that is why the twodimensional log-normal probability distribution is assumed. It is assumed that the dimensionless random variab- les $Y_{1}=\Delta / l, Y_{2}=\omega_{\mathrm{L}} / \omega_{1}$ are not correlated. The $Y_{2}$ domain taken into consideration is truncated to finite values - from zero to the tripled expected value of the variable instead of the whole positive part of the $Y_{2}$-axis. Having in mind this truncation, the value of the total probability equals 0.9999 which can be assumed as 1 . The values of $Y_{1}$ are not truncated. Note that the coefficients of variation $\sigma / \mu$ equal 0.3 thus they are not small.

Table 1. Deterministic characteristics of the considered Ben-Ahin bridge cable (Caetano 2007)

\begin{tabular}{l|c}
\hline Length $l[\mathrm{~m}]$ & 110.5 \\
Cross-sectional area $P\left[\mathrm{~cm}^{2}\right]$ & 82.6 \\
Initial tension force $S[\mathrm{kN}]$ & 4900 \\
Maximal sag $z_{\mathrm{o}}[\mathrm{m}]$ & 0.099 \\
Mass per unit length $m[\mathrm{~kg} / \mathrm{m}]$ & 64.8 \\
The Young modulus $E[\mathrm{GPa}]$ & 210 \\
First eigenfrequency $\omega_{1}[\mathrm{rad} / \mathrm{s}]$ & 7.818 \\
Angle between cable chord and deck $\theta\left[{ }^{\circ}\right]$ & 60 \\
The Irvine parameter $\lambda$ & 0.0727 \\
\hline
\end{tabular}

Assumed characteristics of the random log-normal variables $Y_{1}=\Delta / l \quad[\%]$ and $Y_{2}=\omega_{\mathrm{L}} / \omega_{1}[-]$ are as follows:

- mean values $\mu_{y 1}, \quad \mu_{y 2}$ : respectively 2.280 and 0.051 ,

- standard deviations $\sigma_{y 1}, \sigma_{y 2}: 30 \%$ of each mean value.

Due to (8), the solution $w(t)$ depends on the stochastic process $\Delta(t)=\Delta(t, \gamma)$. Therefore, the maximal in-plane displacement of the cable (in the middle of the cable span) becomes also a stochastic process, $w(t)=w(t, \gamma)$. Deterministic Eqn (8) is linear but it is nonlinear from the stochastic point of view. Random parameters are present in the function of the kinematic excitation as well as in the solution $w(t)$, but not in any explicit form.

The analysis uses conditional probability distributions and conditional moments assuming a sequence of deterministic values of kinematic excitation frequencies $\omega_{\mathrm{L}, i}$. For each fixed $\omega_{\mathrm{L}, i}$, the amplitude of kinematic excitation $\Delta$ is the only one random variable which is considered. It means that Eqn (8) is solved repeatedly for discretized values of the excitation frequencies $\omega_{\mathrm{L}, i}$. Making use of the one-dimensional solutions and their (conditional) probability distributions, random solution of the bi-variate stochastic problem can be found using the formula of total probability (or total moments), like $\mathrm{P}[A]=\sum \mathrm{P}\left[A / \omega_{\mathrm{L}, i}\right] \cdot \mathrm{P}\left[\omega_{\mathrm{L}, i}\right]$. Excitation frequencies $\omega_{\mathrm{L}, i}$ for each step are calculated as the probability mass center for each selected sub-domain (zone). There are 10 selected zones with the same probability $p=0.1$. The idea of this partition is presented in Figure 2, in a simplified case of three zones. 


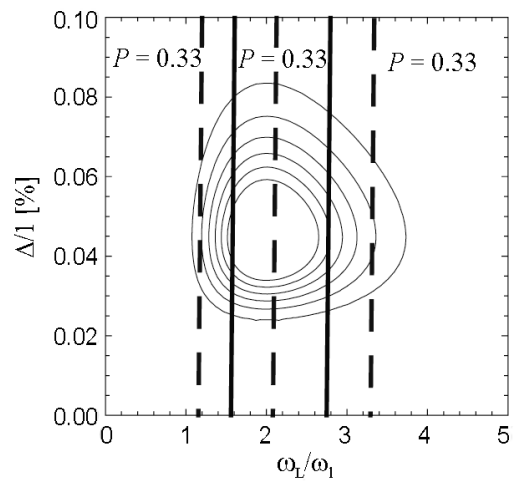

Fig. 2. Example: $Y_{2}$-partition of the density function region into three vertical zones of equal probabilities $Y_{1}=\Delta / l, p=1 / 3$; mass centers are shown for each region (dashed lines)

Ten coordinates of the right end of the selected zones (as in Fig. 2 for three zones - black lines) equal: 1.50, $1.71,1.87,2.03,2.18,2.35,2.55,2.80,3.19,6.84$. Ten coordinates of the mass-center for each selected zone ( $\omega_{\mathrm{L}, i}$, as in Fig. 2 - dashed lines) equal: 1.31, 1.61, 1.79, $1.95,2.11,2.27,2.45,2.67,2.97,3.69$.

\subsection{The polynomial chaos approximation of the log-normal random variable}

The input log-normal variable $Y_{1}=\Delta / l$ can be expressed in the form of the exponential function:

$$
Y_{1}=\exp \left(\mu_{x 1}+\sigma_{x 1} \cdot \xi\right),
$$

where: $\xi$ is a standard Gaussian random variable and $\mu_{x 1} ; \sigma_{x 1}$ are the mean value and the standard deviation of the normal distribution. The parameters $\mu_{x 1}, \sigma_{x 1}$ depend on $\mu_{y 1}, \sigma_{y 1}$, so the characteristics of the normal distribution $\xi$ generating the variable $Y_{1}$ can be expressed as:

$$
\sigma_{x 1}^{2}=\ln \left(1+\left(\sigma_{y 1} / \mu_{y 1}\right)^{2}\right), \quad \mu_{x 1}=\ln \left(\mu_{y 1}\right)-\sigma_{x 1}^{2} / 2 .
$$

For all fixed values of $Y_{2}$ (ten zones with the same probability $p=0.1$ ), the expansion based on the Hermite polynomials is used to approximate the random variable $Y_{1}$ :

$$
Y_{1} \approx \beta_{\Delta} \cdot \mathrm{He}_{0}(\xi)+\beta_{\Delta 1} \cdot \mathrm{He}_{1}(\xi)+\ldots+\beta_{\Delta m} \cdot \mathrm{He}_{m}(\xi),
$$

where $\operatorname{He}_{0}(\xi)=1, \mathrm{He}_{1}(\xi)=\xi, \mathrm{He}_{2}(\xi)=\xi^{2}-1$, etc. (Ghamen, Spanos 1991). The coefficients $\beta_{\Delta i}$ are calculated using the scalar product and the orthogonality of the Hermite polynomials:

$$
\begin{gathered}
\left\langle Y_{1}, \mathrm{He}_{j}(\xi)\right\rangle=\left\langle\sum_{i=0}^{m} \beta_{\Delta i} \cdot \mathrm{He}_{i}(\xi), \mathrm{He}_{j}(\xi)\right\rangle= \\
\sum_{i=0}^{m} \beta_{\Delta i} \cdot \delta_{i j} \cdot i !=\beta_{\Delta j} \cdot j ! \\
\beta_{\Delta j}=\frac{1}{j !} \cdot\left\langle Y_{1}, \operatorname{He}_{j}(\xi)\right\rangle, j=0,1, \ldots, m
\end{gathered}
$$

or in the explicit form:

$$
\begin{aligned}
& \beta_{\Delta j}=\frac{1}{j !} \cdot\left\langle\exp \left(\mu_{x 1}+\sigma_{x 1} \cdot \xi\right), \mathrm{He}_{j}(\xi)\right\rangle= \\
& \frac{1}{j !} \cdot \frac{1}{\sqrt{2 \pi}} \cdot \int_{-\infty}^{\infty} \exp \left(\mu_{x 1}+\sigma_{x 1} \cdot y\right) \cdot \operatorname{He}_{j}(y) \cdot e^{-y^{2} / 2} \mathrm{~d} y .
\end{aligned}
$$

The integration in (13) yields the results $\beta_{\Delta i}$ for the parameters $\mu_{x 1}, \sigma_{x 1}$ :

$$
\begin{aligned}
& \beta_{\Delta 0}=5.100 \cdot 10^{-4}, \beta_{\Delta 1}=1.497 \cdot 10^{-4}, \beta_{\Delta 2}=2.198 \cdot 10^{-5} \\
& \beta_{\Delta 3}=2.150 \cdot 10^{-6}, \beta_{\Delta 4}=1.578 \cdot 10^{-7}, \beta_{\Delta 5}=9.266 \cdot 10^{-9} .
\end{aligned}
$$

\subsection{Solution of the one-dimensional stochastic problem}

Since the random input parameter $Y_{1}$ is in the form of (11), the solution is a stochastic process $w(t, \xi)$ approximated as follows:

$w(t, \xi)=\sum_{i=0}^{\infty} w_{i}(t) \cdot \mathrm{He}_{i}(\xi) \approx \sum_{i=0}^{m} w_{i}(t) \cdot \mathrm{He}_{i}(\xi), \quad m<\infty$.

Generally, in the $m$-dimensional subspace, the PC expansion leads to the approximate equation resulting from (8):

$$
\begin{aligned}
& S_{1} \cdot \sum_{i=0}^{m} \frac{\mathrm{d}^{2}}{\mathrm{~d} t^{2}} w_{i}(t) \cdot \mathrm{He}_{i}(\xi)+S_{2} \cdot \sum_{i=0}^{m} \frac{\mathrm{d}}{\mathrm{d} t} w_{i}(t) \cdot \mathrm{He}_{i}(\xi)+ \\
& \left(S_{3}+S_{4} \cdot\left(\beta_{\Delta 0} \cdot \mathrm{He}_{0}(\xi)+\beta_{\Delta 1} \cdot \mathrm{He}_{1}(\xi)+\ldots+\beta_{\Delta m} \cdot\right.\right. \\
& \left.\mathrm{He}_{m}(\xi)\right) \cdot \cos \left(\omega_{\mathrm{L}} t\right)+S_{5} \cdot\left(\beta_{\Delta 0} \cdot \mathrm{He}_{0}(\xi)+\beta_{\Delta 1} \cdot \mathrm{He}_{1}(\xi)+\right. \\
& \left.\left.\ldots+\beta_{\Delta m} \cdot \mathrm{He}_{m}(\xi)\right)^{2} \cdot \cos \left(\omega_{\mathrm{L}} t\right)^{2}\right) \cdot \sum_{i=0}^{m} w_{i}(t) \cdot \mathrm{He}_{i}(\xi)+ \\
& S_{6} \cdot\left(\beta_{\Delta 0} \cdot \mathrm{He}_{0}(\xi)+\beta_{\Delta 1} \cdot \mathrm{He}_{1}(\xi)+\ldots+\beta_{\Delta m} \cdot \mathrm{He}_{m}(\xi)\right)^{3} . \\
& \cos \left(\omega_{\mathrm{L}} t\right)^{3}+S_{7} \cdot\left(\beta_{\Delta 0} \cdot \mathrm{He}_{0}(\xi)+\beta_{\Delta 1} \cdot \mathrm{He}_{1}(\xi)+\right. \\
& \left.\ldots+\beta_{\Delta m} \cdot \mathrm{He}_{m}(\xi)\right)^{2} \cdot \cos \left(\omega_{\mathrm{L}} t\right)^{2}+S_{8} \cdot\left(\beta_{\Delta 0} \cdot \mathrm{He}_{0}(\xi)+\right. \\
& \left.\beta_{\Delta 1} \cdot \mathrm{He}_{1}(\xi)+\ldots+\beta_{\Delta m} \cdot \mathrm{He}_{m}(\xi)\right) \cdot \frac{\mathrm{d}^{2}}{\mathrm{~d} t^{2}} \cos \left(\omega_{\mathrm{L}} t\right)+ \\
& S_{9} \cdot\left(\beta_{\Delta 0} \cdot \mathrm{He}_{0}(\xi)+\beta_{\Delta 1} \cdot \mathrm{He}_{1}(\xi)+\ldots+\beta_{\Delta m} \cdot \mathrm{He}_{m}(\xi)\right) . \\
& \frac{\mathrm{d}}{\mathrm{d} t} \cos \left(\omega_{\mathrm{L}} t\right)+S_{10} \cdot\left(\beta_{\Delta 0} \cdot \mathrm{He}_{0}(\xi)+\beta_{\Delta 1} \cdot \mathrm{He}_{1}(\xi)+\right. \\
& \left.\ldots+\beta_{\Delta m} \cdot \mathrm{He}_{m}(\xi)\right) \cdot \cos \left(\omega_{\mathrm{L}} t\right)=\varepsilon_{m} \approx 0,
\end{aligned}
$$

where $\omega_{\mathrm{L}}=$ const .

In the approximated approach, a random residuum $\varepsilon_{m}$ should be as small as possible. It is assumed that the residuum is perpendicular to each base vector of the $m$ dimensional subspace, so each scalar product $\left\langle\varepsilon_{m}, \mathrm{He}_{k}(\xi)\right\rangle$ should be zero for $k=0,1, \ldots, m$. Note that only zero element is perpendicular to all base vectors, so 
the approximation becomes better if the subspace dimension $m$ increases. The solution method justifies the term "projection" because the approximate solutions belong to subspaces spanned on the first $m$ base vectors.

The multiplication of both sides of Eqn (15) by the polynomials $\mathrm{He}_{k}(\xi)$ and next the application of the expected value operator to both sides of (15) leads to $(m+1)$-dimensional system of ordinary differential equations (Ghamen, Spanos 1991). In this system, some coefficients $e_{i k m n}, \quad e_{i k m}$ appear, for example $e_{i k m}=\mathrm{E}\left[\mathrm{He}_{i}(\xi) \cdot \mathrm{He}_{k}(\xi) \cdot \mathrm{He}_{m}(\xi)\right]$; they are natural numbers, independent of the considered boundary problem, and they can be obtained by the use of the Gaussian integrals or the MC simulation. The search for the $m+1$ unknown functional coefficients $w_{i}(t)$ can use the standard Runge-Kutta method of the numerical integration available in Mathematica ${ }^{\circledR}$ software. Zero initial conditions are assumed in this situation. Finally, the solution of the random problem can be assembled by multiplication of the obtained functions $w_{0}(t), w_{1}(t), \ldots, w_{m}(t)$ by the orthogonal Hermite polynomials, as in (14).

\subsection{Solution of the two-dimensional stochastic problem}

Bearing in mind the solutions obtained for the onedimensional stochastic problem, it is possible to extend the analysis to the two-dimensional one where both $\Delta$ and $\omega_{\mathrm{L}}$ are random. From a mathematical point of view, such a generalization can use a product-base of the Hermite polynomials, like $B_{\mathrm{ij}}=\operatorname{He}_{i}\left(\xi_{1}\right) \cdot \mathrm{He}_{j}\left(\xi_{2}\right)$ where two generating Gaussian variables are used. Alternatively, a combination of the expansion with a discretization can be also effective. For the estimation of first two moments, the following expression yield from the total probability formula:

$$
\begin{aligned}
\mathrm{E}[w(t)] & =\sum_{i=1}^{n} \mathrm{E}\left[w(t) \mid \omega_{\mathrm{L}, i}\right] \cdot \mathrm{P}\left(\omega_{\mathrm{L}, i}\right), \\
\operatorname{Var}[w(t)] & =\sum_{i=1}^{n} \operatorname{Var}\left[w(t) \mid \omega_{\mathrm{L}, i}\right] \cdot \mathrm{P}\left(\omega_{\mathrm{L}, i}\right),
\end{aligned}
$$

where: $\omega_{\mathrm{L}, i}$ is the $i^{\text {th }}$ fixed kinematic excitation frequency taken with a probability $\mathrm{P}\left(\omega_{\mathrm{L}, i}\right)-$ here, for ten discretization points, it is assumed that $\mathrm{P}\left(\omega_{\mathrm{L}, i}\right)=0.1=$ const $; \quad \mathrm{E}\left[w(t) \mid \omega_{\mathrm{L}, i}\right]$ and $\operatorname{Var}\left[w(t) \mid \omega_{\mathrm{L}, i}\right]$ are conditional moments.

\section{Polynomial chaos expansions - results of the numerical calculations}

Eqn (15) is solved 10 times for the ten deterministic values of $\omega_{\mathrm{L}, i}$, calculated in Section 3.2. Next, the solution for two-dimensional stochastic problem is assembled as in (16). The solutions based on the PC expansions are compared with the results yielding from the direct $\mathrm{MC}$ simulation.

\subsection{Results of the one-dimensional stochastic problem}

The amplitude $\Delta$ of the kinematic excitation is assumed to be the log-normal random variable, frequencies of the kinematic excitation are considered as deterministic values. Solutions - in the form of the variance - are presented in Figure 3 and Figure 4 for two selected excitation frequencies $\omega_{\mathrm{L}}$. The first value $\omega_{\mathrm{L}} \approx 2 \omega_{1}$ corresponds to the resonant vibrations (Fig. 3), the second one presents stable solutions (Fig. 4). The value of $\omega_{1}$ is constant, presented in Table 1. Note that the expected value equals $\mathrm{E}[w(t, \xi)]=\langle w(t, \xi), 1\rangle=\left\langle w(t, \xi), \mathrm{He}_{0}(\xi)\right\rangle=w_{0}(t), \quad$ due to (4) and (14) thus it is not sensitive to the length of the polynomial chaos expansion (parameter $m$ ).

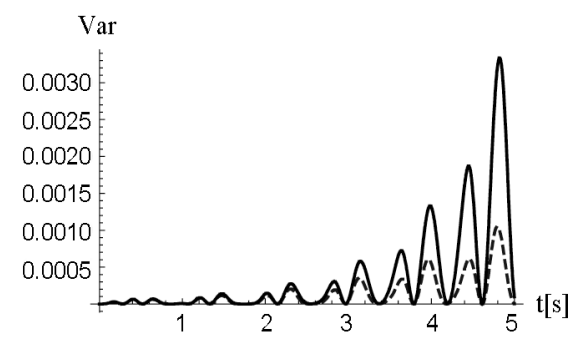

Fig. 3. Variance $\operatorname{Var}[w(t, \xi)]$ in $\left[\mathrm{m}^{2}\right]$ of the solution for $\omega_{\mathrm{L}}=1.95 \omega_{1}$ (inside the resonant zone); black line - solution for the PC expansion with 6 Hermite polynomials $(i \leq 5)$; dashed line - solution for the polynomial chaos expansion with 2 Hermite polynomials $(i \leq 1)$

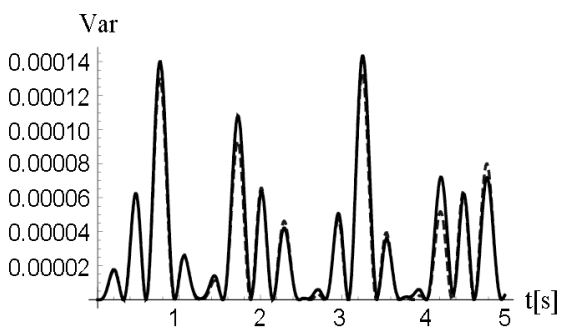

Fig. 4. Variance $\operatorname{Var}[w(t, \xi)]$ in $\left[\mathrm{m}^{2}\right]$ of the solution for $\omega_{\mathrm{L}}=1.61 \omega_{1}$ (outside the resonant zone); black line - solution for the PC expansion with 6 Hermite polynomials $(i \leq 5)$; dashed line - solution for the polynomial chaos expansion with 2 Hermite polynomials $(i \leq 1)$

For some excitation frequencies, a strong amplification of vibrations in the time domain appears, i.e. for the excitation frequencies close to the doubled natural eigenfrequency of the cable $\omega_{1}$ (the instability zone). For that case the solution based on the 2-term approximation strongly differ from the one based on the 6-term approximation (Fig. 3).

However, a much better convergence is observed for a stable solution (Fig. 4), so the linear stochastic PC approximation ( $i \leq 1)$ is sufficient but only far away from the resonant frequency (Fig. 4). For resonant vibrations, a more extended expansion is required, compared to the case of stable solutions. 


\subsection{Results of the two-dimensional stochastic problem}

The first two moments of the two-dimensional stochastic problem for the random variables $\omega_{\mathrm{L}}$ and $\Delta$ are calculated with the use of (16) according to the pre-selected ten values of $\omega_{\mathrm{L}, i}$.

The evaluation of the expected value $\mu=\mathrm{E}[w(t, \xi)]$ does not depend on the expansion length $m$; again, the variance convergence rate does depend on $m$ - Figure 5 .

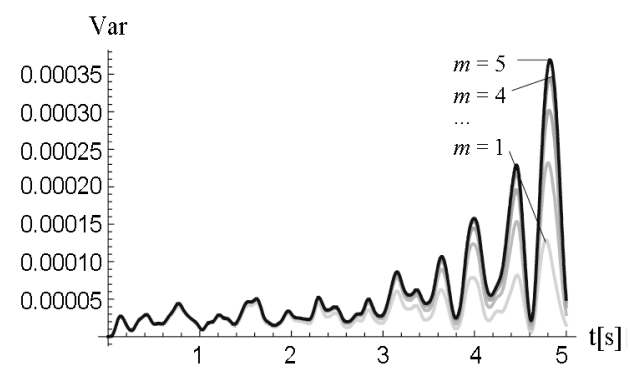

Fig. 5. Variance $\operatorname{Var}[w(t, \xi)]$ in $\left[\mathrm{m}^{2}\right]$ of the two-dimensional stochastic problem; from the black line - solution for the PC expansion with 6 Hermite polynomials, to very fair gray line solution for the PC expansion with 2 Hermite polynomials

\section{Monte Carlo simulation - validation of the results based on the polynomial chaos approximation}

The results of the numerical calculations yielding from the PC approximation are checked using the MC simulation for both situations presented in the paragraph 4, i.e. for one and two log-normal input variables, respectively. The first six Hermite polynomials are used $(m=5)$.

\subsection{Validation of the one-dimensional stochastic problem}

For the first case, excitation frequency is far away from the resonant zone, so the stable vibrations are analyzed for $\omega_{L}=1.31 \omega_{1}$ (Fig. 6). The second case refers to the resonant vibrations, excitation frequency is close to the doubled natural frequency of the system, $\omega_{L}=2.11 \omega_{1}$ (Fig. 7).

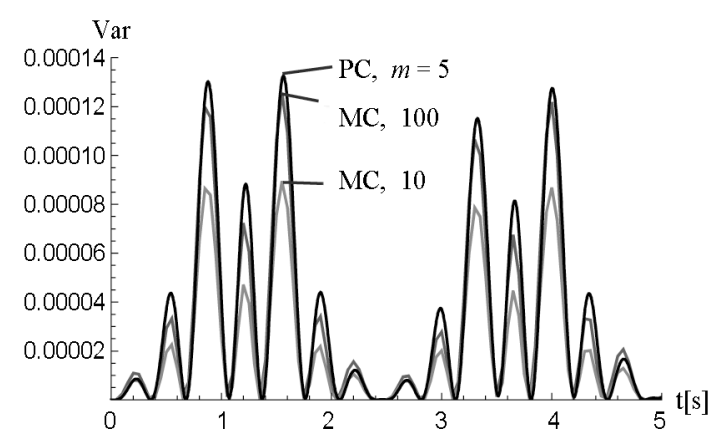

Fig. 6. Variance Var $\left[\mathrm{m}^{2}\right]$ of the solution of the one-dimensional problem for stable vibrations; black line - solution obtained using the PC expansion with 6 Hermite polynomials; from fair gray line - solution obtained using the MC simulation with 10 simulations to dark gray line - solution obtained using MC simulation with 100 simulations

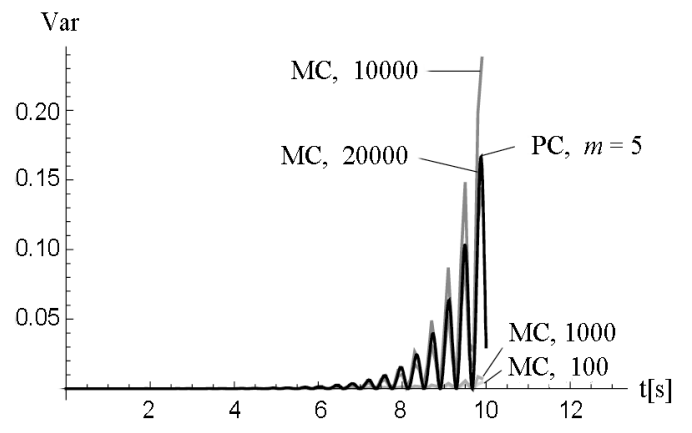

Fig. 7. Variance Var $\left[\mathrm{m}^{2}\right]$ of the solution of the one-dimensional problem for resonant vibrations; black line - solution obtained using the PC expansion with 6 Hermite polynomials; from fair gray line - solution obtained using the MC simulation with 100 simulations to dark gray line - solution obtained using the MC simulation with 20000 simulations

\subsection{Validation of the two-dimensional stochastic problem}

The variance of the solutions obtained by the use of the $\mathrm{PC}$ approximation and the MC simulations is presented in Figure 8.

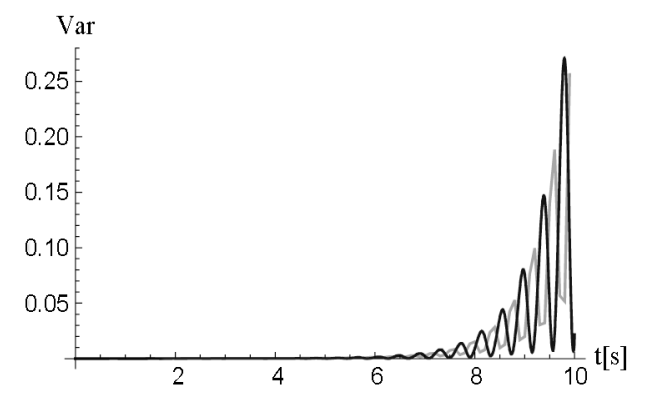

Fig. 8. Variance Var $\left[\mathrm{m}^{2}\right]$ of the solution for the twodimensional problem; black line - solution obtained using the PC expansion with 6 Hermite polynomials; gray line - solution obtained using the MC simulation with 20000 simulations

From the qualitative point of view, both solutions $\operatorname{Var}=\operatorname{Var}(t)$ in Figure 8 reveal the same tendency. For both, the PC and the MC results, the variance strongly increases in time. Phase shifting between the solutions may be caused by the different approaches used for describing the random character of the variable $\omega_{\mathrm{L}}$, i.e. by a discretization technique (a continuous spectrum of the excitation frequencies is considered in the MC simulation).

In the presented example, the $\mathrm{PC}$ expansion is more efficient (faster) than the MC simulation. A good convergence of the variance is reached for 20000 MC simulations, compared to only 6 polynomials in the PC expansion. For the solutions outside the resonant zone, a better convergence of the PC approximation is still more evident.

\section{Summary and general conclusions}

The parametric vibrations belong to one of the less recognized parts of the traditional structural dynamics. However, the parametric resonance is often reported as a 
potentially significant danger, mostly in bridge cables. The projection method based on the finite dimensional polynomial subspace can be an effective alternative to popular perturbation methods, the method of moments or to the Monte Carlo simulation.

The presented examples proved that the polynomial chaos expansion is more efficient (or simply faster) than the Monte Carlo simulation, especially if the resonant vibrations appear. Furthermore, the advantage of the polynomial chaos expansion is obvious if the random variables of interest (including the response variables) are Gaussian or close to the Gaussian distribution. Indeed, a very small number of expansion terms is required, say $m \sim 1 \div 2$. On the other hand, the Monte Carlo simulation is less insensitive to probability distributions of the input variables as well as the increasing number of the input random variables. Furthermore, the numerical implementation of the Monte Carlo simulation is also much easier.

The used conditional probabilities and conditional moments can reduce the dimension of the model (number of considered random variables) with no significant loose of accuracy. However, the expansion method is also effective for multidimensional spaces with polynomial product bases. Note also that it is much easier to obtain results in the form of the probability histograms, by making use of the pre-calculated deterministic $w_{\mathrm{i}}(t)$ and the Monte Carlo simulations - applied this time only to $\mathrm{He}_{i}(\xi)$.

\section{References}

Anders, M.; Hori, M. 1999. Stochastic finite element method for elasto-plastic body, International Journal for Numerical Methods in Engineering 46(11): 1897-1916. http://dx.doi.org/10.1002/(SICI)1097-0207(19991220)46: $11<1897:$ :AID-NME758>3.0.CO;2-3

Blatman, G.; Sudret, B. 2010. An adaptive algorithm to build up sparse polynomial chaos expansions for stochastic finite element analysis, Probabilistic Engineering Mechanics 25(2): 183-197.

http://dx.doi.org/10.1016/j.probengmech.2009.10.003

Blatman, G.; Sudret, B. 2011. Adaptive sparse polynomial chaos expansion based on least angle regression, Journal of Computational Physics 230(6): 2345-2367. http://dx.doi.org/10.1016/j.jcp.2010.12.021

Brzakala, A. 2013. Application of the polynomial chaos approximation to a stochastic parametric vibrations problem, Archives of Civil and Mechanical Engineering 13(2): 220-228. http://dx.doi.org/10.1016/j.acme.2012.12.007

Caetano, E. 2007. Cable vibrations in cable-stayed bridges. Structural Engineering Documents 9, International Association for Bridge and Structural Engineering IABSE.

Crestaux, T.; Le Matre, O.; Martinez, J.-M. 2009. Polynomial chaos expansion for sensitivity analysis, Reliability Engineering and System Safety 94(7): 1161-1172. http://dx.doi.org/10.1016/j.ress.2008.10.008

El Ouni, M. H.; Ben Kahla, N. 2012. Nonlinear dynamic analysis of a cable under first and second order parametric excitations, Journal of Civil Engineering and Management 18(4): 557-567. http://dx.doi.org/10.3846/13923730.2012.702994

Georgakis, C. T.; Taylor, C. A. 2005. Nonlinear dynamics of cable stays. Part I: sinusoidal cable support excitation,
Journal of Sound and Vibration 281(3-5): 537-564.

http://dx.doi.org/10.1016/j.jsv.2004.01.022

Ghamen, R. G.; Spanos, P. D. 1991. Stochastic finite elements: a spectral approach. New York: Springer-Verlag. 214 p.

Ghanem, R. G.; Spanos, P. D. 1993. Stochastic Galerkin expansion for nonlinear random vibration analysis, Probabilistic Engineering Mechanics 8(3-4): 255-264. http://dx.doi.org/10.1016/0266-8920(93)90019-R

Irvine, M. 1981. Cable structures. New York: Dover Publications, Inc. 259 p.

Le Maitre, O.; Najm, H.; Ghanem, R.; Knio, O. 2004. Multiresolution analysis of Wiener-type uncertainty propagation schemes, Journal of Computational Physics 197(2): 502-531. http://dx.doi.org/10.1016/j.jcp.2003.12.020

Lilien, J. L.; Pinto Da Costa, A. 1994. Vibration amplitudes caused by parametric excitation of cable stayed structures, Journal of Sound and Vibration 174(1): 69-90. http://dx.doi.org/10.1006/jsvi.1994.1261

Lucor, D.; Karniadakis, G. 2004. Predictability and uncertainty in flow-structure interactions, European Journal of Mechanics B/Fluids 23(1): 41-49.

http://dx.doi.org/10.1016/j.euromechflu.2003.05.001

Manolis, G.; Karakostas, C. 2003. A Green's function method to SH-wave motion in random continuum, Engineering Analysis with Boundary Elements 27(2): 93-100. http://dx.doi.org/10.1016/S0955-7997(02)00086-3

Nayfeh, A. H.; Mook, D. T. 1995. Nonlinear oscillations. New York: John Wiley\&Sons. 720 p.

Qu, D.; Xu, C. 2013. Generalized polynomial chaos decomposition and spectral methods for the stochastic Stokes equations, Computers \& Fluids 71: 250-260. http://dx.doi.org/10.1016/j.compfluid.2012.10.001

Royer-Carafangi, G. F. 2003. Parametric-resonance-induced vibrations in network cable-stayed bridges. A continuum approach, Journal of Sound and Vibration 262(5): 11911222. http://dx.doi.org/10.1016/S0022-460X(02)01149-5

Sarkar, A.; Ghanem, R. G. 2002. Mid-frequency structural dynamics with parameter uncertainty, Computer Methods in Applied Mechanics and Engineering 191(47-48): 5499-5531. http://dx.doi.org/10.1016/S0045-7825(02)00465-6

Sudret, B. 2008. Global sensitivity analysis using polynomial chaos expansions, Reliability Engineering and System Safety 93(7): 964-979. http://dx.doi.org/10.1016/j.ress.2007.04.002

Takahashi, K. 1991. Dynamic stability of cables subjected to an axial periodic load, Journal of Sound and Vibration 144(2): 323-330. http://dx.doi.org/10.1016/0022-460X(91)90752-6

Wan, X.; Karniadakis, G. 2005. An adaptive multi-element generalized polynomial chaos method for stochastic differential equations, Journal of Computational Physics 209(2): 617-642. http://dx.doi.org/10.1016/j.jcp.2005.03.023

Wan, X.; Karniadakis, G. 2006. Long-term behavior of polynomial chaos in stochastic flow simulations, Computer Methods in Applied Mechanics and Engineering 195(4143): 5582-5596. http://dx.doi.org/10.1016/j.cma.2005.10.016

Xiu, D.; Karniadakis, G. 2002a. Modelling uncertainty in steady-state diffusion problems via generalized polynomial chaos, Computer Methods in Applied Mechanics and Engineering 191(43): 4927-4948.

http://dx.doi.org/10.1016/S0045-7825(02)00421-8 
Xiu, D.; Karniadakis, G. 2002b. Stochastic modelling of flowstructure interactions using generalized polynomial chaos, Journal of Fluids Engineering 124(1): 51-59. http://dx.doi.org/10.1115/1.1436089

Xiu, D.; Karniadakis, G. 2003. A new stochastic approach to transient heat conduction modelling with uncertainty, International Journal of Heat and Mass Transfer 46(24): 4681-4693.

http://dx.doi.org/10.1016/S0017-9310(03)00299-0
Zhou, Q.; Nielsen, S. R. K.; Qu, W. L. 2006. Semi-active control of three-dimensional vibration of an inclined sag cable with magnethorheological dampers, Journal of Sound and Vibration 296(1-2): 1-22.

http://dx.doi.org/10.1016/j.jsv.2005.10.028

Wlodzimierz BRZAKALA. DSc, PhD, Eng, Associate Professor at the Wroclaw University of Technology, Faculty of Civil Engineering. His research interests focus on structural safety and uncertainty modeling, geoengineering, numerical methods.

Aneta HERBUT. PhD, Eng, Assistant Professor at the Wroclaw University of Technology, Faculty of Civil Engineering. Her research interests include structure dynamics; active, passive and semi-active vibration mitigation methods; cable dynamics for deterministic and stochastic cases; stochastic differential equations. 